\title{
Evaluación de tres técnicas para el manejo de conducta odontológica en pacientes con trastorno del espectro autista
}

\section{Evaluation of three techniques for the dental behavior management in autistic spectrum disorder patients}

ISSN-L 1560-9111; eISSN: 1609-8617

Artículo Original

Maritza Perales-Terán 1,a, Aida Sabbagh-Haddad 2,b, Karla Isabel Juárez-Ibarra ${ }^{1, c}$, Norma Cruz-Fierro 1,d

${ }^{1}$ Universidad Autónoma de Nuevo León, Facultad de Odontología, Monterrey Nuevo León, México.

2 Universidade de São Paulo, Faculdade de Odontologia,

Sao Paulo, Brasil.

${ }^{a}$ Maestría en Odontología Avanzada.

${ }^{\circ}$ Doutora em Diagnóstico Bucal.

' Maestría en Odontología Avanzada.

${ }^{d}$ Doctor en Filosofía con Orientación en Psicología.

\section{Correspondencia:}

Norma Cruz-Fierro: norma.cruzfr@uanl.edu.mx Facultad de Odontología Universidad Autónoma de Nuevo León. Dr. Eduardo Aguirre Pequeño. Col. Mitras Centro. Monterrey Nuevo León, México.64460. ORCID: 0000-0001-8910-388X

\section{Coautores:}

Maritza Perales-Terán: maritzaperales20@gmail.com ORCID: 0000-0001-8353-884X

Aida Sabbagh-Haddad: aidasabbagh@hotmail.com ORCID: 0000-0001-6769-8480

Karla Isabel Juárez-Ibarra: karla.juarezibr@uanl.edu.mx ORCID: 0000-0003-0856-9827

\section{Editor:}

Yuri Castro-Rodríguez

Universidad Nacional Mayor de San Marcos, Perú.

Conflicto de intereses: los autores declaran que no existe ningún tipo de conflictos de interés.

Fuente de financiamiento: autofinanciado

Recibido: 03/12/20

Aceptado: 13/01/21

Publicado: 15/02/21 dental, sin embargo en la técnica DMH se observó respuesta corporal-conductual que refleja tensión emocional.

Palabras clave: Trastorno del espectro autista; Trastornos sensoriales; Cuidado Dental; Trastornos de la conducta; Control del comportamiento (fuente: DeCS BIREME).

\begin{abstract}
Objective. Evaluate three behavior management techniques: Systematic Desensitization (SD), the Treament and Education of Autistic and related Communication Handicapped Children (TEACCH) and Tell, Show, Do (TSD) Method, during dental care for people with Autism Spectrum Disorder (ASD). Methods. Eighteen people from Northeast Mexico diagnosed with autism spectrum disorder participated, with a range of 13 to 15 years, divided in to three groups, according to the TEACCH, SD and TSD technique with six participants per group. A dental prophylaxis was performed on the participants, using the behavior management and communication technique according to the group, the behavior was evaluated using the Frankl's behavior rating scale (FBRS) and Comfort Behavior Scale (CBS). Chi square, ANOVA and Student's t tests were used to compare the results. Results. Behavior evaluated with Frankl scale was definitely positive in TEACCH (33.3\%) and DS (16.7\%) groups. Similar positive behavior in the three groups $(66,7 \%)$. Similar negative behavior in DS and DMH (16.7\%). Definitively negative behavior only in the DMH group (16.7\%). With CBS scale, only the DMH group
\end{abstract}

(C) Los autores. Este artículo es publicado por la revista Odontología Sanmarquina de la Facultad de Odontología, Universidad Nacional Mayor de San Marcos. Este es un artículo de acceso abierto, distribuido bajo los términos de la licencia Creative Commons Atribucion - No Comercia_Compartir Igual 4.0 Internacional. (http://creativecommons.org/licenses/by-nc-sa/4.0/) que permite el uso no comercial, distribución y reproducción en cualquier medio, siempre que la obra original sea debidamente citada. 
presented increased muscle tone $(p=0.013)$ and evident not sustained tension in some facial muscles $(p=0.001)$, when compared with TEACCH and DS by ANOVA analysis. Conclusions. The three techniques evaluated are effective for managing behavior of people with ASD during dental treatment, however, in DMH technique, definitely negative behavior and a corporal-behavioral response were observed, reflecting emotional stress.

Keywords: Autism spectrum disorder; Sensory disorders; Dental care; Behavior disorders; Behavior Control (source: MeSH NLM).

\section{Introducción}

La atención dental a personas con trastorno del espectro autista (TEA) es frecuente por su alta incidencia de caries, gingivitis, enfermedad periodontal y perdida de piezas dentales ${ }^{1}$. El daño causado por estas patologías es mayor debido al hábito de retener los alimentos en la boca, la ingesta de alimentos blandos y ricos en carbohidratos, y las limitaciones para la higiene bucal caracteristicos en ellos ${ }^{2}$, aumentando la complejidad de los tratamientos, estas circunstancias junto con los problemas conductuales, hacen necesario que el odontólogo recurra a protocolos de manejo de conducta.

La prevalencia del TEA, a nivel mundial es de uno en cada 160 personas ${ }^{3}$, las cifras varian entre paises y continentes, en México población incluida en el presente estudio, la prevalencia es uno en 115 niños, esto significa que casi el $1 \%$ de los niños en México, alrededor de 400000 , tienen TEA ${ }^{4}$. El diagnostico de este trastorno heterogéneo del neurodesarrollo se realiza en las primeras etapas de la vida y persiste en la edad adulta.

Las personas con TEA tienen características fisiológicas y cognitivo-conductuales especificas, su conducta varía según el deterioro al coeficiente intelectual $(\mathrm{CI})$, este se clasifica en deterioro severo con CI por debajo del promedio, deterioro leve con CI en nivel normal y TEA de alto funcionamento con CI por encima del promedio ${ }^{5}$. Esta información ayuda a determinar la autonomía del paciente para su autocuidado, a categorizar su conductua y establecer protocolos para una adecuada comunicación.

De acuerdo con las características de su neurodesarrollo, presentan alteraciones en la comunicación, la interacción social, restricción de intereses, dificultad de integración sensorial, patrones repetitivos de comportamiento y falta de cooperación ${ }^{6,7}$. Y responden diferente a los estímulos sensoriales ${ }^{8}$, situación desafiante para el odontólogo ya que en una consulta dental, existe una sobre estimulación sensorial por los sonidos, la luz y el movimiento del equipo dental, el sabor y olor de los materiales dentales, la presencia del odontólogo y personal de apoyo, que puede afectar el desarrollo de la atención dental ${ }^{9}$. Además, la mayoría de estos pacientes presentan trastornos de ansiedad ${ }^{10}$.

Las limitaciones del procesamiento de la información sensorial se pueden reforzar usando técnicas para el control conductual, estableciendo una adecuada comunicación y disminuyendo las emociones negativas durante la atención dental en personas con y sin TEA ${ }^{11}$. En esta categoria de métodos se incluyen las técnicas Desensibilización Sistemática (DS) ${ }^{12,13}$, el método TEACCH (Treament and Education of Autistic and related Communication Handicapped Children) ${ }^{14,15}$ y Decir, Mostrar, Hacer ${ }^{16}$. Y fueron seleccionadas para este trabajo por sus resultados efectivos, como el método TEACCH, usado en preescolares con TEA, en el hogar y la escuela redujo los síntomas de autismo y sus conductas desadaptativas 17. La técnica DS, con mayor uso en psicología, para controlar trastornos de ansiedad y una gran variedad de fobias ${ }^{13}$.

Estas técnicas se han usado también en odontología, sin embargo, la técnica Decir, Mostrar, Hacer, tiene una mayor trayectoria de uso sobretodo en odontopediatría para el control de la ansiedad desde la primera cita, con excelentes resultados sobre todo al usarla en conjunto con métodos de distracción visual como aplicaciones en el celular para educar al paciente desde su casa ${ }^{18}$. Partiendo de la hipótesis que la estructuración sistemática es un refuerzo efectivo para el aprendizaje y adaptación de las personas con TEA, el propósito de este trabajo fue evaluar tres técnicas para el manejo de conducta durante la atención dental en adolescentes con diagnóstico de TEA. Los resultados obtenidos contribuyen en ampliar la información que se tiene de su uso en la consulta dental en esta población.

\section{Métodos}

Participantes. Se seleccionó una muestra no probabilística incidental de 18 personas con TEA, alumnos de una institución del Noreste de México, la invitacion a participar la realizó un odontólogo en una junta de padres de familia. Los participantes se dividieron en tres grupos (seis personas cada uno) según la técnica: TEACCH, DS y DMH. Como criterio de inclusión se consideró, el diagnostico de TEA, la edad entre 13 a 15 años, integrados a un sistema educativo, familiarizados en escuchar y seguir instrucciones ${ }^{19}$, con respuesta a estímulos sensoriales bucodentales dentro del promedio. Fueron excluidos los participantes cuyo padre o tutor no firmaron el consentimiento informado, dejaron alguna pregunta sin respuesta, o no participaron en las dos actividades del proyecto.

Procedimiento. Un odontólogo atendió a los participantes de febrero a junio del 2019. La participación se desarrolló en dos etapas, en la primera se explicó a los padres de familia los detalles del área de trabajo, características de la clínica, material, instrumental y la técnica de manejo de conducta según el grupo. Y contestaron el 
cuestionario de datos generales del paciente y la escala de sensibilidad bucodental. En la segunda etapa se realizó la profilaxis dental utilizando la técnica de manejo de conducta según el grupo. La conducta se evaluó al inicio y al final de la profilaxis usando la escala de Frankl ${ }^{20}$. Los cambios corporales durante la cita se evaluaron con la escala Comfort Behavior Scale ${ }^{21}$.

Técnica TEACCH: Treament and Education of Autistic and related Communication Handicapped Children ${ }^{22}$, es un método desarrollado para trabajar en personas con TEA, basado en la enseñanza visual estructurada usa pictogramas que indican que se debe de hacer, el orden para hacerlo y cuándo finaliza, aprovechando la fortaleza visual del paciente con TEA ${ }^{23,24}$. Se realizaron pictogramas basados en los pictogramas "Yo voy al dentista" y "El dentista, limpia mis dientes" ${ }^{25}$. Durante la entrevista el odontólogo explicó el procedimiento al paciente, apoyado con los pictogramas, iniciando el proceso de familiarización paciente-odontólogo, paciente-clínica, paciente-instrumento, paciente-sonidos, paciente- personal de apoyo. En la segunda etapa se realizó la profilaxis dental apoyado con los pictogramas.

Técnica Desensibilización Sistemática (DS): Es un método usado para modificar la conducta en el tratamiento de fobias ${ }^{12} \mathrm{y}$ determinar respuestas de ansiedad y conductas de evitación en trastornos ansiosos. La técnica explica que al exponer a una persona a un estímulo condicionado desaparecerá la respuesta de ansiedad ${ }^{13}$. Utiliza imágenes y videos para exponer al paciente a estímulos visuales, auditivos, olfatorios o táctiles que le generen ansiedad o fobia. En odontología, se ha usado para reducir la ansieded dental en niños con TEA con buenos resultados ${ }^{26}$. Se realizaron imágenes y videos de los posibles estímulos asociados con ansiedad y fobia durante una profilaxis dental. En la entrevista el odontólogo expuso al paciente a estos estímulos, y realizó una explicación verbal. En la etapa de profilaxis dental se utilizaron como refuerzo las imágenes y videos.

Técnica Decir, Mostrar, Hacer (DMH): Es un método de primera elección para el manejo de conducta en niños en odontología, para anticiparle las sensaciones que experimentará durante el proceso y lograr que el paciente permita que se usen los instrumentos y materiales en el, evitando el comportamiento no deseado, temor o resistencia al tratamiento. El método utiliza comunicación verbal, no verbal y refuerzo positivo ${ }^{27}$. En la primera etapa el odontólogo explicó verbalmente el procedimiento a realizar durante la profilaxis dental. En la segunda etapa se realizó el tratamiento usando los recursos Decir: explicando el procedimiento, Mostrar: enseñando al paciente los aspectos visuales, auditivos, olfativos y táctiles del procedimiento, Hacer: realizando y finalizando el procedimiento de forma continua, sin interrumpir la explicación ni la demostración ${ }^{28}$.

\section{Instrumentos de evaluación:}

Escala de Sensibilidad bucodental: Dirigida a padres de familia o cuidadores tiene un tiempo de respuesta de 10 a 15 minutos. Esta escala se diseño para evaluar la sensibilidad bucodental en personas con TEA, e identificar las conductas negativas que influyen en el manejo del entorno de la clínica dental. Se fundamenta en cuestionarios de reactividad sensorial general ${ }^{29-31}$. La escala tiene validez de contenido por juicio de expertos de $90 \%$. Contiene 31 preguntas y 5 factores: F-1 sensibilidad a sabores y consistencias. F-2 sensibilidad al cepillado dental. F-3 sensibilidad a la atención dental, F-4 sensibilidad al sillón dental y F-5 sensibilidad al medio ambiente del consultorio dental. Con cuatro opciones de respuestas tipo Likert que va de $0=$ nunca a $3=$ Siempre. La puntuación alta indica alto nivel de sensibilidad ${ }^{32}$.

Frankl's behavior rating scale (FBRS) ${ }^{20}$, es una escala de observación y calificación. Su versión en español, es usada por el odontólogo para evaluar la conducta de nińos asignando de 1 a 4 puntos según el tipo de conducta observada: Tipo 1. Definitivamente negativo. Paciente con rechazo definitivo al tratamiento, muestra llanto enérgico, miedo o cualquier evidencia de negativismo extremo. Tipo 2.- Negativo. Paciente renuente a aceptar el tratamiento, poco cooperador, con alguna actitud negativa pero no extrema al tratamiento. Tipo 3.- Positivo. Paciente con aceptación positiva al tratamiento, unas veces con curiosidad y voluntad otras con reserva de seguir instrucciones del dentista. Tipo 4.- Definitivamente Positivo. El paciente con relación buena y positiva con el dentista, con interés en los procedimientos dentales, riendo y disfrutando de la situación ${ }^{33}$.

Comfort Behavior Scale es la versión modificada de la escala Comfort Scale, desarrollada para evaluar dolor y distrés en niños hospitalizados ${ }^{21}$. Esta escala se ha usado en personas con y sin discapacidad ${ }^{34,35}$, y ha sido traducida en diferentes idiomas, entre ellos el español y validada principalmente en pacientes pediátricos ${ }^{36,37}$. En esta escala un observador analiza y califica la intensidad de siete manifestaciones conductuales: vigilia, grado de agitación/calma, llanto, respuesta respiratoria, movimiento físico, tono muscular y expresión facial, asigna una puntuación de 1 a 5 puntos. La suma total de menor valor es 6 puntos y la más alta de 30 puntos. El punto de corte para diferenciar presencia de dolor/ distrés en el paciente es mayor a 17 puntos.

El presente estudio siguió lo establecido en el Reglamento de la ley General de Salud en Materia de Investigación para la Salud ${ }^{38}$, y el código de conducta de la American Psychological Association (APA) ${ }^{39}$. Fue revisado y autorizado por el comité de bioética de la Facultad de Odontología de la Universidad Autónoma de Nuevo León/ México, Número de Folio: 00137 SPSI- 010613.

Análisis estadístico. Los datos se analizaron con el software estadístico SPSS-21, para la escala de sensibilidad bucodental se analizó su consistencia interna por alfa de Cronbach, estadística descriptiva y comparación de resultados calculando el Chi cuadrado. Para las puntuaciones de la escala de Frankl obtenidas al iniciar y al finalizar la cita de la profilaxis dental, se analizó la frecuencia y porcentaje de la conducta observada por grupo usando una tabla de contingencia y Chi cuadrado. 
Con la escala Comfort Behavior Scale se realizó una medición final que incluía expresiones corporales de conductas observadas durante el procedimiento. Se evaluó valores de tendencia central y para comparar las respuestas de la conducta entre grupos se usó análisis de ANOVA, confirmado la distribución con la prueba de Kruscal Wallis. La diferencia entre grupos con t de Student y prueba de Levene.

\section{Resultados}

En este estudio participaron 18 personas con TEA, 13 hombres y cinco mujeres, con edades entre 13 a 15 años. La escala de sensibilidad bucodental evaluada con el coeficiente alfa de Cronbach, mostró consistencia interna adecuada $\alpha=0,779$. Los resultados del análisis descriptivo de la escala de sensibilidad bucodental, analizado por subescalas en los tres grupos, mostró valores de media semejantes (Tabla 1). Para la compación de los resultados se calculó el Chi cuadrado, no se observó significancia estadística $(p>0,05)$.
Los datos obtenidos con la escala de Frankl, al iniciar y al finalizar el procedimiento dental, se analizaron usando una tabla de contingencia para revisar la frecuencia y porcentaje de la conducta observada por grupo y se calculó el Chi cuadrado entre lo observado en al inicio y al finalizar el tratamiento (Figura 1). El resultado de la la escala de Frankl al final del procedimiento fue definitivamente positiva en los grupos TEACCH $(33,3 \%)$ y DS $(16,7 \%)$. Conducta positiva semejante en los tres grupos (66,7\%). Conducta negativa en DS y DMH (16,7\%). Conducta definitivamente negativa en el grupo $\mathrm{DMH}$ $(16,7 \%)$, en la comparación de los resultados se observó diferencia estadisticamente significativa $p=0,0308$.

El resultado de los seis parámetros conductuales de la escala Comfort Behavior Scale (CBS) se reporta en la tabla 2. Para la comparación de respuesta física entre los grupos se realizó el análisis ANOVA observando diferencia estadísticamente significativa en los resultados Tono Muscular $(p=0,013)$ y Tensión Facial $(p=0,001)$. La

Tabla 1. Estadísticos descriptivos de las subescalas de factores del cuestionario de sensibilidad, por grupo de manejo de conducta

\begin{tabular}{|c|c|c|c|c|c|c|c|c|c|}
\hline \multirow[t]{2}{*}{ Subescala } & \multicolumn{2}{|c|}{$\begin{array}{c}\text { TEACCH } \\
n=6\end{array}$} & \multicolumn{2}{|c|}{$\begin{array}{l}\mathrm{DS} \\
\mathrm{n}=6\end{array}$} & \multicolumn{2}{|c|}{$\begin{array}{c}\text { DMH } \\
n=6\end{array}$} & \multicolumn{2}{|c|}{$\begin{array}{l}\text { Total } \\
\mathrm{n}=18\end{array}$} & \multirow{2}{*}{$\frac{\chi^{2}}{p}$} \\
\hline & $\mathrm{M}$ & D.E. & M & D.E. & M & D.E. & M & D.E. & \\
\hline Sabor/Consistencia & 9,83 & 2,63 & 12,66 & 4,13 & 10,16 & 4,53 & 10,88 & 3,84 & 0,522 \\
\hline Cepillado Dental & 9,00 & 2,60 & 10,00 & 2,28 & 10,16 & 2,13 & 9,72 & 2,27 & 0,354 \\
\hline Atención Dental & 6,66 & 6,65 & 6,00 & 4,38 & 6,00 & 1,89 & 6,22 & 4,45 & 0,220 \\
\hline Sillón Dental & 2,83 & 5,23 & 2,33 & 3,88 & 3,33 & 3,55 & 2,83 & 4,04 & 0,401 \\
\hline Medio ambiente & 5,50 & 1,87 & 5,83 & 213 & 3,50 & 2,07 & 4,94 & 2,18 & 0,646 \\
\hline
\end{tabular}

TEACCH: Treatment and Education of Autistic and related Communication Handicapped Children / DS: Desensibilización Sistemática/ DMH: Decir, Mostrar, Hacer/ $\chi^{2}$ : Chi cuadrado. M: media / D.E.: desviación estándar

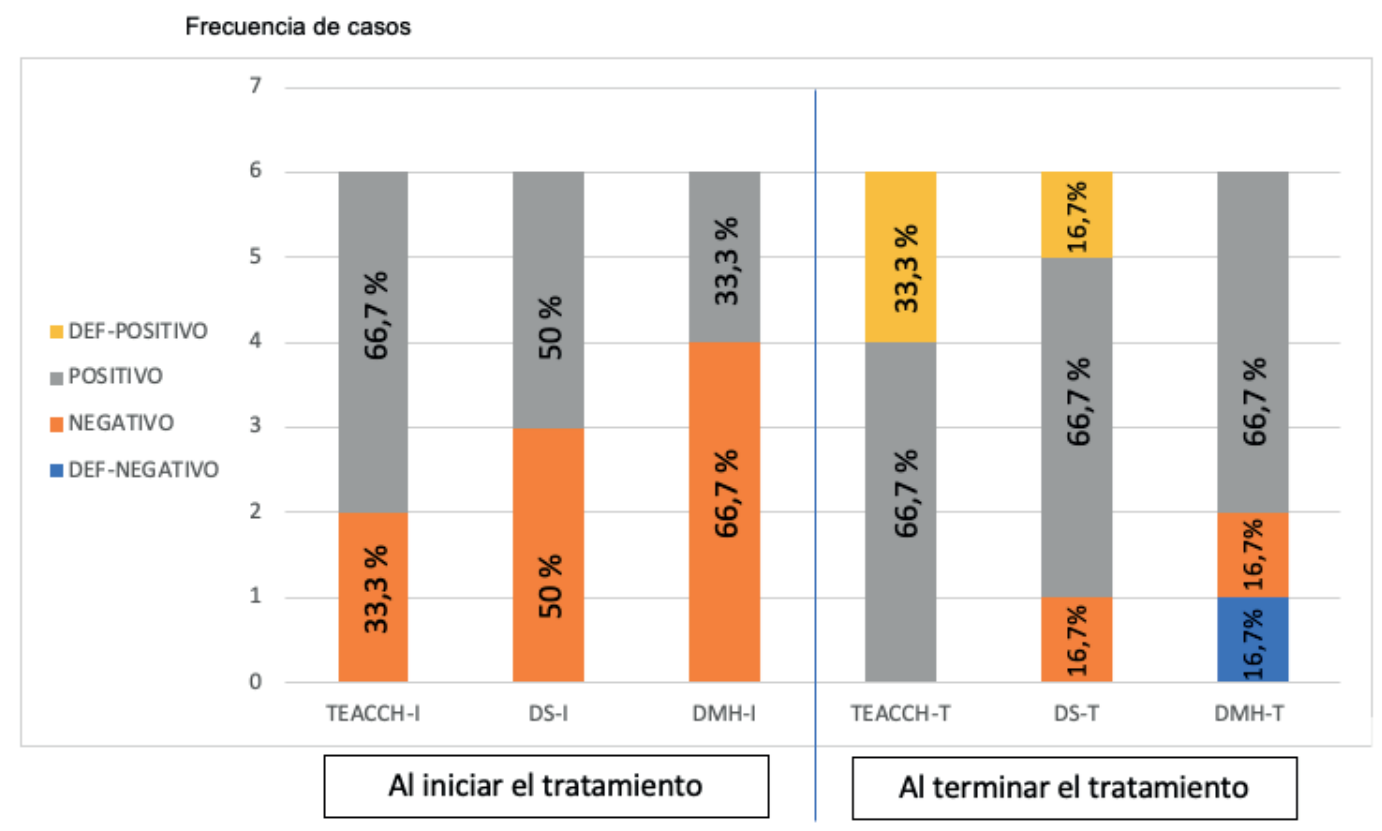

Figura 1. Frecuencia del tipo de conducta según la escala de Frankl, por grupo de manejo de conducta

TEACCH: Treatment and Education of Autistic and related Communication Handicapped Children / DS: Desensibilización Sistemática/ DMH: Decir, Mostrar, Hacer. 
Tabla 2. Distribución de respuesta de la escala Comfort Behavior Scale, entre los tres grupos

\begin{tabular}{|c|c|c|c|c|c|c|c|}
\hline & & \multicolumn{3}{|c|}{ Técnicas } & \multicolumn{2}{|c|}{ ANOVA } & \multirow{2}{*}{$\begin{array}{c}\text { Kruskal-Wallis } \\
\mathrm{p}\end{array}$} \\
\hline & & TEACCH & DS & DMH & $F$ & $p$ & \\
\hline \multirow[t]{5}{*}{ Vigilancia } & Profundamente Dormido & 1 & 0 & 0 & 0,407 & 0,673 & 0,581 \\
\hline & Ligeramente dormido & 0 & 1 & 1 & & & \\
\hline & Somnolencia & 2 & 4 & 2 & & & \\
\hline & Despierto & 3 & 1 & 2 & & & \\
\hline & Hiper-alerta & 0 & 0 & 1 & & & \\
\hline \multirow[t]{5}{*}{ Calma-Agitación } & Calma & 0 & 0 & 2 & 0,068 & 0,934 & 0,968 \\
\hline & Un poco nervioso & 4 & 4 & 1 & & & \\
\hline & Ansioso & 2 & 1 & 2 & & & \\
\hline & Muy Ansioso & 0 & 1 & 1 & & & \\
\hline & Pánico & 0 & 0 & 0 & & & \\
\hline \multirow[t]{5}{*}{ Respuesta Respiratoria } & No respiración espontánea & 0 & 2 & 1 & 0,512 & 0,608 & 0,593 \\
\hline & Respiración espontánea & 5 & 3 & 3 & & & \\
\hline & Inquietud o resistencia & 1 & 1 & 2 & & & \\
\hline & Respira activamente/ Tose & 0 & 0 & 0 & & & \\
\hline & Agitación respiratoria & 0 & 0 & 0 & & & \\
\hline \multirow[t]{5}{*}{ Llanto } & No hay sonidos de llanto & 2 & 2 & 2 & 0,151 & 0,860 & 0,909 \\
\hline & Llanto ocasional/gemidos & 4 & 4 & 3 & & & \\
\hline & Lloriqueo & 0 & 0 & 1 & & & \\
\hline & Llorando & 0 & 0 & 0 & & & \\
\hline & Gritando o chillando & 0 & 0 & 0 & & & \\
\hline \multirow{5}{*}{$\begin{array}{l}\text { Movimiento } \\
\text { Físico }\end{array}$} & Sin Movimiento & 1 & 3 & 2 & 0,159 & 0,853 & 0,980 \\
\hline & Movimiento leve ocasional & 5 & 0 & 3 & & & \\
\hline & Movimiento leve frecuente & 0 & 2 & 0 & & & \\
\hline & Mov. vigoroso extremidades & 0 & 1 & 1 & & & \\
\hline & Mov. vigoroso cabeza-torso & 0 & 0 & 0 & & & \\
\hline \multirow[t]{5}{*}{ Tono Muscular } & Sin tono muscular & 2 & 2 & 0 & 8,076 & 0,013 & 0,014 \\
\hline & Disminución tono muscular & 4 & 2 & 1 & & & \\
\hline & Tono muscular normal & 0 & 2 & 2 & & & \\
\hline & Aumento del tono muscular & 0 & 0 & 3 & & & \\
\hline & Rigidez muscular extrema & 0 & 0 & 0 & & & \\
\hline \multirow[t]{5}{*}{ Tensión Facial } & Músculos faciales relajados & 0 & 0 & 0 & 10,00 & 0,001 & 0,007 \\
\hline & Tono normal de la cara & 6 & 6 & 2 & & & \\
\hline & Tensión facial no sostenida & 0 & 0 & 4 & & & \\
\hline & Tensión facial sostenida & 0 & 0 & 0 & & & \\
\hline & Músculos faciales contraídos & 0 & 0 & 0 & & & \\
\hline
\end{tabular}

TEACCH: Treatment and Education of Autistic and related Communication Handicapped Children // DS: Desensibilización Sistemática/ DMH: Decir, Mostrar, Hacer

prueba de Kruscal Wallis confirma que la distribución de las conductas Tono Muscular $\left(\mathrm{X}^{2}=8,426, p=0,014\right)$ y Tensión Facial $\left(X^{2}=9,714, p=0,007\right)$ es diferente entre los grupos.

Para comparar las técnicas TEACCHI DMH; DS/ DMH y TEACCH/DS y analizar la diferencia se utilizó la prueba t de Student (Tabla 3). Observando en las técnicas TEACCHy DS diferencia estadísticamente significativa en los resultados Tono Muscular y Tensión Facial al compararlas con la técnica DMH respectivamente.

\section{Discusión}

El presente trabajo tuvo como objetivo evaluar las técnicas TEACCH, DS y DMH en pacientes con TEA, para el manejo de conducta durante la atención dental, Debido a que el desarrollo convencional del tratamiento dental es influido por las diferentes alteraciones de estas persona, se evaluó la respuesta a los estimulos con la escala de sensibilidad bucodental, y se determinó un nivel de sensibilidad semejante en los grupos, con mayor informe de antecedentes de sensibilidad a 
Tabla 3. Prueba de Levene y $t$ de Student de la escala CBS, entre las técnicas

\begin{tabular}{lccc}
\hline & TEACCH / DMH & DS / DMH & \multicolumn{2}{c}{ TEACCH / DS } \\
$\mathbf{p}$ & $\mathbf{p}$ & 0,765 \\
\hline Vigilancia & 0,614 & 0,341 & 0,687 \\
Calma-Agitación & 1,000 & 0,787 & 0,363 \\
Respuesta Respiratoria & 1,000 & 0,461 & 1,000 \\
Llanto & 0,664 & 0,664 & 0,570 \\
Movimiento Físico & 0,734 & 0,817 & 0,448 \\
Tono Muscular & 0,002 & 0,022 & 0,765 \\
Tensión Facial & 0,025 & 0,025 & 2 \\
\hline
\end{tabular}

TEACCH: Treatment and Education of Autistic and related Communication Handicapped Children / DS: Desensibilización Sistemática/ DMH: Decir, Mostrar, Hacer

sabores, consistencias y al cepillado dental, y menor sensibilidad a los movimientos del sillón dental y luz del equipo dental.

Estos resultados son semejantes a lo informado por Stein et al ${ }^{40}$ quienes evaluaron hiporeacitividad sensorial al tratamiento dental en personas con TEA de 2 a 18 años, observaron sensibilidad a sonidos y sabores, hiporeacitividad sensorial a la luz de la lámpara, ruidos en la sala de espera y a nuevas sensaciones de tacto y gusto ${ }^{40}$. Es importante mencionar que la respuesta sensorial también es influida por el desarrollo psicosocial y su inclusión en los diferentes entornos, y experiencias previas que lo familiaricen con el cuidado dental en casa o en un consultorio dental.

Para el manejo de la conducta del paciente se han propuesto diferentes abordajes conductuales que mejoran los principales sintomas del TEA ${ }^{41}$. En el presente trabajo se eligieron las técnicas TEACCH, DS y DMH, y para evaluar la conducta observada en cada técnica se usó la escala de Frankl, observando mejores resultados en la técnica TEACCH al presentar conducta positiva y definitivamente positiva en los 6 pacientes. En comparación con la Técnica DS con un paciente con respuesta negativa y la técnica DMH con un paciente con respuesta negativa y uno definitivamente negativa.

La técnica DMH, se ha usado en odontología con pacientes pediátricos con y sin discapacidad. Radhakrishna et al ${ }^{42}$ compararon tres técnicas de modificación de conducta: Decir Mostrar PlayDoh, Juego del dentista en el celular y DMH tradicional, para controlar la ansiedad durante un procedimiento de operatoria dental. Los resultados de las escalas de Frankl y FLACC (Cara, pierna, actividad, llanto, consolación por sus siglas en inglés). Muestran menor eficacia de la técnica DMH tradicional, resultado que coincide con este trabajo, al observar conducta negativa y definitivamente negativa en este grupo.

La DS es una técnica utilizada en odontología para el tratamiento de fobias y trastornos de ansiedad en personas con TEA. Algunos protocolos presentan mejor resultado al usar la técnica DS sola, que combinada con benzodiacepinas ${ }^{43}$ y también se ha observado que la DS mejora al combinarla con técnicas de relajación ${ }^{44}$. La técnica TEACCH usada para diagnóstico bucal ${ }^{45}$ favoreció la conducta de pacientes con TEA. Resultado que coincide con los observados en este trabajo, y en intervenciones que usan fotografías o imágenes digitales para facilitar a personas con TEA su adaptación a un medio ambiente.

Los protocolos para el manejo de conducta recomiendan evaluar el llanto, expresiones faciales, quejas, movimientos del cuerpo, ya que los cambios en estas conductas son indicador de dolor, ansiedad o distrés y un signo objetivo para reforzar las técnicas y evitar una mala experiencia y mala conducta en citas posteriores, ${ }^{16} \mathrm{en}^{\mathrm{este}}$ trabajo se evaluó la respuesta corporal por medio de la escala Comfort Behavior Scale CBS, observando mayor tono muscular y tensión facial en el grupo $\mathrm{DMH}$.

Evaluar la respuesta del paciente con TEA a diferentes entornos es un procedimiento complejo pero necesario para conocer las necesidades individuales que faciliten su adaptación al medio ambiente odontológico. Dentro de la limitaciones encontradas en la literatura es la complejidad para controlar las variables que influyen en la conducta y que representan posibilidad de sesgo en la medición y en la estandarización de las conductas. Como el nivel de inclusión del paciente, la respuesta individual a estimulos sensoriales, las experiencias previas de familiarización con el cuidado dental en casa y en la consulta dental, el uso previo y dominio de una técnica de manejo conductual. Se recomienda en futuras investigaciones, el trabajo multidisciplinario con un psicólogo para fortalecer el diseño, control de las variables cognitivas y evaluación de la intervención e incluir mayor número de citas, analizando la evolución a largo plazo con cada técnica.

Con los resultados obtenidos al evaluar tres técnicas para el manejo de conducta odontológica en pacientes con TEA, se concluye que las tres técnicas evaluadas $T E$ $A C C H$, DS y DMH son efectivas, sin embargo la técnica TEACCH presentó mejores resultados en el proceso de comunicación efectiva y refuerzo de adaptación al medio ambiente al observar conductas positivas y definitivamente positivas en este grupo. Confirmando que la estructuración sistemática y el uso de ayudas visuales y 
auditivas para el seguimiento de instrucciones, son técnicas de gran ayuda para la comunicación y adaptación del paciente con TEA al medioambiente odontológico.

\section{Agradecimiento}

Los autores agradecen el apoyo de la Maestría en Odontología Avanzada de la Universidad Autónoma de Nuevo León, a la Asociación Regiomontana de Niños Autistas (ARENA) y a la Academia Interamericana de Odontología para Pacientes Especiales (AIOPE), por su apoyo.

\section{Referencias bibliográficas}

1. Naidoo M, Singh S. The Oral health status of children with Autism Spectrum Disorder in KwaZulu-Nata, South Africa. BMC Oral Health. 2018;18(1):165. DOI:10.1186/s12903-018-0632-1.

2. Campos C, Sabbagh-Haddad A. Transtornos de comportamento e tratamento odontológico. En: Sabbagh-Haddad, A. Odontologia para Pacientes com Necessidades Especiais. São Paulo: Santos, 2007.

3. World Health Organization, meeting report (WHO). Autism spectrum disorders \& other developmental disorders, from raising awareness to building capacity. 2013. [Consultado el 24 de Julio 2020]. Accesible en: http://www.who.int/mental_health/maternalchild/autism_report/en/

4. Fombonne E, Marcin C, Manero A, Bruno R, Diaz C, Villalobos M, et al. Prevalence of Autism Spectrum Disorders in Guanajuato, Mexico: The Leon survey. J Autism Dev Disord. 2016;46(5):1669-1685. DOI: 10.1007/s10803-016-2696-6

5. Gomes P, Lima L, Bueno M, Araújo L, Souza N. Autism in Brazil: a systematic review of family challenges and coping strategies. J Pediatr. 2015;91(2):111-121. http:// dx.doi.org/10.1016/j.jped.2014.08.009

6. American Psychiatric Association. Publication diagnostic and statical manual of mental disorders. DSM- 5. London: British Library; 2013.

7. Tayama M, Tateno M, Park TW, Ukai W, Hashimoto E, Saito T. The study of cognitive characteristics in Asperger's disorder by using a modified prisoner's dilemma game with a variable payoff matrix. Plos One. 2012;7(11):48794.

8. Tomchek S, Dunn W. Sensory processing in children with and without autism: a comparative study using the short sensory profile. Am J Occup 2016;61(2):190-200.

9. Suarez, M. Sensory processing in children with autism spectrum disorders and impact on functioning. Pediatr Clin North Am. 2012;203-214.

10. Zaboski B, Storch E. Comorbid autism spectrum disorder and anxiety disorders: A brief review. Future Neurol. 2018;13(1):31-37.

11. Sabbagh-Haddad AS. Odontologia para pacientes com necessidades especiais. São Paulo: Livraria Santos Editora Ltda; 2007.

12. Wolpe J. Psicoterapia por inhibición recíproca. Bilbao, España: Desclée de Brouwer; 1981.
13. Vallejo-Slocker L, Vallejo M. Sobre la desensibilización sistemática. Una técnica superada o renombrada. Acción psicol. 2016;13(2):157-168. http://dx.doi.org/10.5944/ ap.13.2.16539

14. Shopler E, Mesibov G, Hearsey K. Structured teaching in the TEACCH system. En Schopler E, Mesibov GB, editores. Current issues in autism. Learning and cognition in autism. 1a. ed. New York. Plenum Press; 1994. p. 243-268. DOI: 10.1007/978-1-4899-1286-2_13

15. University of North Carolina. TEACCH ${ }^{\triangleright}$ Autism Program. 2020, [Consultado el 19 de Octubre 2020]. Accesible en: https://teacch.com/resources/

16. American Academy of Pediatric Dentistry AAPD. Behavior guidance for the pediatric dental patient. Pediatr Dent. 2020;292-310. Accesible en: https://www.aapd. org/globalassets/media/policies_guidelines/bp_behavguide.pdf

17. D'Elia L, Valeri G, Sonnino F, Fontana I, Mammone A, Vicari S. A longitudinal study of the TEACCH program in different settings: the potential benefits of low intensity intervention in preschool children with Autism Spectrum Disorder. J Autism Dev Disord. 2014;44(3):615626. DOI: $10.1007 / \mathrm{s} 10803-013-1911-\mathrm{y}$

18. Elicherla S, Bandi S, Nuvvula S, Challa R, Saikiran K, Priyanka V. Comparative evaluation of the effectiveness of a mobile app (Little Lovely Dentist) and the tellshow-do technique in the management of dental anxiety and fear: a randomized controlled trial. J Dent Anesth Pain Med. 2019;19(6):369-378. DOI: 10.17245/ jdapm.2019.19.6.369.

19. Frankl S, Shire F, Fogels H. Should the parent remain with the child in the dental operatory? J Dent Chil. 1962;29:150-62.

20. Van Dijk M, Peters J, Van Deventer P, Tibboel D. The Comfort Behavior Scale: a tool for assessing pain and sedation in infants. Am J Nurs. 2005;105(1):33-36.

21. Pérez Acevedo S, Dávila Marrero E. Perfil neurocognitivo de jóvenes diagnosticados con el Trastorno del Espectro del Autismo altamente funcional. Rev Puertorriq Psicol. 2019;30(1):156-168.

22. Schopler E, Brehm S, Kinsbourne M, Reichle RJ. The effect of treatment structure on development of autistic children. Arch Gen Psychiat.1971;24:415-421.

23. Zink A, Diniz M, Rodrigues M. Use of a picture exchange communication system for preventive procedures in individuals with autism spectrum disorder: pilot study. Spec Care Dentist. 2016;36(5):254-9. DOI: 10.1111/ scd.12183.

24. Welterlin A, Turner-Brown L, Harris S, Mesibov G, Delmolino L. The home TEACCHing program for toddlers with autism. J Autism Dev Disord. 2012;42(9):1827-35.

25. Ariz, A. (2014). Metodología de aprendizaje: Método Teacch. Maestra especial PT. [Consultado el 2 de Febrero 2019]. Accesible en: http://www.autismonavarra.com/ wp-content/uploads/2014/03/Dentista.pdf

26. Gómez B, Badillo V, Martínez E, Planells P. Intervención odontológica en niños con autismo. La desensibilización sistemática. Científica dental. 2009;6(3):207-215. 
27. American Academy of Pediatric Dentistry AAPD. Guideline on behavoir guidance for the pediatric dental patient. Pediatric Dentistry. 2015;37(5):180-193. [Consultado el 20 de Febrero 2019]. Disponible en: https:// www.aapd.org/globalassets/media/policies_guidelines/ bp_behavguide.pdf

28. Calero I, Aristizabal L, Villavicencio J. Manejo y comportamiento de la nińez temprana en la práctica odontológica. Estomatología. 2012;20(1):45-49.

29. Elwin Teaching M, Schröder A, Ek L, Kjellin L. Development and pilot validation of a sensory reactivity scale for adults with high functioning autism spectrum conditions: Sensory Reactivity in Autism Spectrum (SR-AS). Nord J Psychiatry. 2016;70(2):103-10. DOI: 10.3109/08039488.2015.1053984.

30. Tavassoli T, Hoekstra R, Baron-Cohen S. The Sensory Perception Quotient (SPQ): development and validation of a new sensory questionnaire for adults with and without autism. Mol Autism. 2014;5:29. DOI: 10.1186/2040-2392-5-29

31. The Southwest Autism Research \& Resource Center (SARRC). Autism dental information guide for dental professionals. Versión en español. 2019. [Consultado el 2 de Febrero 2019]. Accesible en: https://www.autismcenter.org

32. Perales-Terán M, Cruz-Fierro N, Vanegas-Farfano $M$, De la Garza-Ramos M, Martínez-González G., Saénz S. Content validity by experts of the questionnaire of sensory response to the odontological environment stimulus in autism spectrum disorder.2020; [Artículo en preparación].

33. Ramos K, Alfaro L, Tirado L, González F. Habilidades sociales y conducta en niños durante la consulta Odontologica. Rev Fac Odont Univ Antioq. 2015;27(1):86107.

34. Narayan V, Samuel S. Appropriateness of various behavior rating scales used in pediatric dentistry: A Review. J Global Oral Health. 2019;2(2):112-117. DOI: 10.25259/JGOH_64_2019

35. Valkenburg A, Boerlage A, Ista E, Duivenvoorden H, Tibboel D, van Dijk M. The COMFORT-behavior scale is useful to assess pain and distress in 0- to 3-year-old children with Down syndrome. Pain. 2011;152(9):20592064. DOI:10.1016/j.pain.2011.05.001

36. Comfort assessment. COMFORT behavior scale, versión en español. 2020. [Consultado el 2 de Marzo 2020]. Accesible en: http://www.comfortassessment.nl/web/index.php/instruments/comfort/
37. Bosch-Alcarazab A, Jordan I, Guàrdia Olmos J, Falcó-Pegueroles A. Adaptación transcultural y características de la versión española de la escala COMFORT Behavior Scale en el paciente crítico pediátrico. Med Intensiva. 2020;44(9):542-550. https://doi.org/10.1016/j.medin.2019.07.001

38. Reglamento de la ley general de salud en materia de investigación para la salud 2014. [Consultado el 20 de Febrero 2019]. Accesible en: http://www.diputados.gob. $\mathrm{mx} /$ LeyesBiblio/regley/Reg_LGS_MIS.pdf

39. American psychological association. Ethical principles of psychologists and code of conduct. The American Ps ychological Association 2017. [Consultado el 20 de Febrero 2019]. Accesible en: https://www.apa.org/ethics/ code/ethics-code-2017.pdf

40. Stein L, Polido J, Cermak S. Oral care and sensory over-responsivity in children with Autism Spectrum Disorders. Pediatr Dent. 2013;35(3):230-235.

41. Ospina M, Krebs J, Clark B, Karkhaneh M, Hartling L, Tjosvold V, et al. Behavioural and developmental interventions for Autism Spectrum Disorder: A clinical systematic review. PLoS ONE. 2018;3(11):e3755. DOI:10.1371/journal.pone.0003755

42. Radhakrishna S, Srinivasan I, Setty J, Krishna M, Melwani A, Hegde K. Comparison of three behavior modification techniques for management of anxious children aged 4-8 years. J Dent Anesth Pain Med 2019;19(1):2936. https://doi.org/10.17245/jdapm.2019.19.1.29

43. Coldwell S, Wilhelm F, Milgrom P, Prall C, Getz T, Spadafora $\mathrm{A}$, et al. Combining alprazolam with systematic desensitization therapy for dental injection phobia. J Anxiety Disord. 2007;21(7):871-887. http://dx.doi.or$\mathrm{g} / 10.1016 /$ j.janxdis.2007.01.001

44. Neacsu V, Sfearcu I, Maru N, Dumitrache M. Relaxation and systematic desensitization in reducing dental anxiety. Social and Behavioral Sciences. 2014;127:474478. http://dx.doi.org/0.1016/j.sbspro.2014.03.293

45. Orellana L, Martínez S, Silvestre F. Training adults and children with an autism spectrum disorder to be compliant with a clinical dental assessment using a TEACCH-Based approach. J Autism Dev Disord. 2014;44:776-785. DOI 10.1007/s10803-013-1930-8 